\title{
Saxagliptin protects against diabetic nephropathy by inhibiting caspase 3/PARP-1-dependent nephrocyte apoptosis
}

\author{
XIAOWEI XING ${ }^{1}$, SHUANG GUO ${ }^{2}$, YUSHENG LIU ${ }^{1}$, JIANGYING KUANG ${ }^{1}$, \\ ZHIWEI HUANG ${ }^{3}$, XIN WANG $^{1}$ and QINGHUA LU ${ }^{1}$ \\ Departments of ${ }^{1}$ Cardiology and ${ }^{2}$ Gastroenterology, The Second Hospital, \\ Cheeloo College of Medicine, Shandong University; ${ }^{3}$ Department of Hematology, \\ The Qilu Children's Hospital of Shandong University, Jinan, Shandong 250033, P.R. China
}

Received June 25, 2019; Accepted June 8, 2021

DOI: $10.3892 / \mathrm{etm} .2021 .10422$

\begin{abstract}
Saxagliptin (SAX) can protect against tissue damage caused by diabetic nephropathy. However, whether this compound can restore kidney function, and its specific mechanism of action remain unclear. The present study explored the therapeutic effects and mechanisms of SAX. Male Wistar rats (8 weeks old) were randomly divided into the following groups: A control group $(n=10)$; a group with streptozocin-induced diabetes mellitus (DM) treated with saline $(n=20)$; and a group with streptozocin-induced DM treated with SAX $(n=20)$. Following 20 weeks of treatment, renal function and the extent of renal damage were assessed based on histological staining using hematoxylin and eosin, periodic acid-Schiff and Masson's trichrome staining. The experimental results indicated that Streptozocin induction of DM led to thicker basement membranes in mesangial cells and a more abundant extracellular matrix. These changes were ameliorated following treatment with SAX. The data demonstrated that renal tissue and renal cell apoptosis were ameliorated significantly following treatment with SAX. Furthermore, the expression levels of the apoptotic genes poly (ADP-ribose) polymerase-1 (PARP-1) and caspase 3 were significantly decreased following treatment with SAX. Therefore, SAX may reduce the extent of renal apoptosis and pathological outcomes in diabetic nephropathy by downregulating the expression of caspase 3 and PARP-1 in the death receptor pathway of apoptosis.
\end{abstract}

Correspondence to: Professor Qinghua Lu or Professor Xin Wang, Department of Cardiology, The Second Hospital, Cheeloo College of Medicine, Shandong University, 247 Beiyuan Big Avenue, Jinan, Shandong 250033, P.R. China

E-mail: luqinghua126@yeah.net; happy97101@126.com

Key words: saxagliptin, caspase 3, PARP-1, diabetic nephropathy, apoptosis

\section{Introduction}

Diabetes can cause various complications, including diabetic nephropathy $(1,2)$, which can result in end-stage renal disease, and requires kidney dialysis or transplant (3-5). Diabetic nephropathy is characterized by glomerular basement membrane thickening and glomerular or tubulointerstitial sclerosis (5). The latter is further characterized by fibrosis in its final stage, and its progression is similar to other progressive kidney diseases (6).

The apoptotic process serves as an important role in the progression of diabetic nephropathy due to production of reactive oxygen species induced by glucose (7-11). Apoptosis is accompanied by the activation of caspase 3 leading to DNA fragmentation and cleavage of protein substrates, including the DNA repair enzyme poly (ADP-ribose) polymerase-1 (PARP-1). PARP-1 serves as an essential role in diabetes and diabetic complications (12). PARP-1 inhibition or deficiency ameliorates nephropathy in $\mathrm{db} / \mathrm{db}^{-/-}$mice with type 2 diabetes (13) and in streptozocin-induced diabetic nephropathy (14). High glucose (HG) levels or hyperglycemia cause activation of the $\mathrm{Bcl} 2 /$ caspase/PARP signaling pathway and stimulates the induction of apoptosis, primarily in proximal tubular cells (15-17).

Dipeptidyl peptidase-4 (DPP-4) is an enzyme that is abundantly expressed in the intestines, kidney, brain, heart and other tissues, and is further activated in diabetic animal models $(6,18)$. As the kidneys contain the highest levels of DPP-4 within the body, which quickly degrade natural glucagon-like peptide (GLP-1), DPP-4 contributes to diabetic nephropathy. This aggravates proteinuria, glomerulosclerosis, and tubulointerstitial fibrosis as a result (19). DPP-4 inhibitors have been shown to improve brain function by reducing mitochondrial dysfunction, insulin resistance, inflammation and apoptosis (20). Treatment with vildagliptin and metformin resulted in the maintenance of the Mini-Mental State Examination score, thus showing a protective role on cognitive functioning compared with treatment with metformin alone (20). DPP-4 inhibition has extrapancreatic protective effects against diet-induced adipose tissue inflammation and hepatic steatosis $(21,22)$. The DPP-4 inhibitor linagliptin increases GLP-1 activity and attenuates oxidative stress-related 
glomerulopathy (23). Combined treatment with linagliptin and inhibitors of the renin-angiotensin-aldosterone system reduces renal dysfunction in type 2 diabetes (24). Other DPP-4 inhibitors have also demonstrated protective effects against diabetic nephropathy, including sitagliptin (25) and gemigliptin, which exert anti-apoptotic effects (26).

The DPP-4 inhibitor saxagliptin (SAX) has been used for the treatment of renal, heart, pancreatic and retinal disorders (27-29). Therefore, the present study investigated whether SAX could exert therapeutic effects on the kidney tissues of a rat model of streptozocin-induced diabetes and whether these effects were accompanied by inhibition of apoptosis.

\section{Materials and methods}

Animals. All animal protocols were reviewed and approved by the Animal Care and Use Committee of Shandong University. For the animal experiments, 8-week old male Wistar rats were purchased from the Experimental Animal Center of Shandong University and maintained with a 12-h day/night cycle. Following 1 week of acclimatization, the animals were randomly alloca ted into a control group $(n=10)$, a diabetes mellitus group $(\mathrm{DM})(\mathrm{n}=20)$, or a $\mathrm{DM}+\mathrm{SAX}$ group $(\mathrm{DM}+\mathrm{SAX})(\mathrm{n}=20)$. DM was induced in the latter two groups via intraperitoneal injection of $65 \mathrm{mg} / \mathrm{kg}$ streptozocin (Sigma-Aldrich; Merck KGaA) solution dissolved in citric acid buffer (30). The diagnosis of diabetes was based on a blood glucose level measurement $>16.7 \mathrm{mmol} / 1$. Following induction of diabetes, mice were monitored for 1 week, and subsequently the control and DM animals were provided saline, whereas the DM + SAX animals were administered SAX (1 mg/kg per day) by gavage. Prior to administration, SAX was prepared in a mortar and dissolved in saline. Following 20 weeks of feeding, the animals were sacrificed using $\mathrm{CO}_{2}$ (the $\mathrm{CO}_{2}$ displacement rate was 20\%), and the kidney and blood samples were collected. During the procedures, 2 rats were used to practice intraperitoneal injection, and three rats were used to practice blood and tissue collection, and these rats were not included in the final analysis. The blood glucose levels (after $6 \mathrm{~h}$ of fasting) were measured three times, prior to injection of STZ, and at 10 and 20 weeks after STZ injection. Urinary and blood samples was collected prior to rats sacrifice.

The determination of urinary albumin excretion was performed following urine collection for $16 \mathrm{~h}$ from rats placed in individual metabolic cages (Nalgene Nunc). Urinary albumin was measured using a specific ELISA kit (cat. no. CSB-E12991r; Cusabio Technology LLC). Blood urea nitrogen (BUN) levels were determined using an ELISA kit (cat. no. FT-D234159; Shanghai FanTai Jinghua Biotechnology Co., Ltd.) and serum creatinine (Scr) levels were determined using Creatinine Companion (cat. no. 1012; Exocell). Blood glucose was measured following $6 \mathrm{~h}$ of fasting using a Glucometer Elite device (Roche Diagnostics). The animals were sacrificed after 30 weeks old, when significant signs of albuminuria and mesangial expansion were evident.

Reagents and antibodies. Antibodies against caspase 3 (cat no. 9662S), cleaved-caspase 3 (cat. no. 9664T), PARP-1 (cat. no. 9542), cleaved-PARP1 (cat. no. 94885S),
Bcl-2 (cat. no. 3498S), Bax (cat. no. 2772T), and $\beta$-actin (cat. no. 4970S) were purchased from Cell Signaling Technology, Inc. Horseradish peroxidase-conjugated goat anti-rabbit IgG (cat. no. A0208) antibody was purchased from Beyotime Institute of Biotechnology and a DAB chromogenic reagent kit was purchased from EMD Millipore. The staining kits for Hematoxylin \& Eosin (H\&E), Masson's, periodic acid-Schiff (PAS) and Sirus red were purchased from Beyotime Institute of Biotechnology.

$H \& E$ staining. The tissues were fixed in $10 \%$ phosphate-buffered formalin solution for $24 \mathrm{~h}$ at room temperature, embedded in paraffin and sectioned at a thickness of $4 \mu \mathrm{m}$ (liver) or $6 \mu \mathrm{m}$ (kidney). The tissue sections were then deparaffinized for $30 \mathrm{~min}$ at room temperature and stained with $\mathrm{H} \& \mathrm{E}$ for $5 \mathrm{~min}$ at room temperature.

Cell culture. HK2 cells were cultured in RPMI-1640 (Gibco; Thermo Fisher Scientific, Inc.) containing $11.2 \mathrm{mmol} / 1$ glucose and $2 \mathrm{mmol} / \mathrm{l} \mathrm{L}$-glutamine at $37^{\circ} \mathrm{C}$ with $5 \% \mathrm{CO}_{2}$. The media was supplemented with $10 \%$ FBS (Hyclone; Cytiva), 100 unit/ml penicillin (Gibco; Thermo Fisher Scientific, Inc.) and $100 \mu \mathrm{g} / \mathrm{ml}$ streptomycin (Gibco; Thermo Fisher Scientific, Inc.). HK2 cells were plated in 6-well plates 1 day before use.

Hochest 33258 staining. HK2 cells were treated with $40 \mathrm{mM}$ $\mathrm{HG}, 40 \mathrm{mM} \mathrm{HG}$ and $10 \mu \mathrm{M} \mathrm{SAX}(\mathrm{HG}+\mathrm{SAX}), 40 \mathrm{mM} \mathrm{HG}$ and $1 \mathrm{mM}$ 3-Aminobenzamide (3-ABA) $(\mathrm{HG}+3-\mathrm{ABA})$ or $40 \mathrm{mM}$ $\mathrm{HG}, 1 \mathrm{mM} 3-\mathrm{ABA}$ and $10 \mu \mathrm{M}$ SAX $(\mathrm{HG}+3-\mathrm{ABA}+\mathrm{SAX})$. The cells were fixed with $4 \%$ polyoxymethylene at room temperature for $30 \mathrm{~min}$, washed twice with PBS, incubated with $10 \mu \mathrm{g} / \mathrm{ml}$ Hochest 33258 (Merck \& Company, Inc.) for $5 \mathrm{~min}$ at room temperature and washed with PBS three times. The cells were observed using a fluorescence microscope (magnification, $\mathrm{x} 20$ ).

Masson, PAS and Sirus red staining. The kidney tissues were excised, fixed using $4 \%$ neutral buffered formalin overnight at $4^{\circ} \mathrm{C}$, embedded in paraffin and sectioned at a thickness of $4 \mu \mathrm{m}$. Masson, PAS and Sirus red staining were performed according to the manufacturer's protocols, and imaged at x20 magnification using a light microscope.

Immunohistochemistry. Tissue sections were blocked using 5\% Donkey serum (cat. no. SL050; Beijing Solarbio Science \& Technology Co., Ltd.; diluted in PBS) for $30 \mathrm{~min}$ at $4{ }^{\circ} \mathrm{C}$, and incubated with antibodies against caspase 3 (1:100), PARP-1 (1:100), or $\beta$-actin $(1: 200)$ overnight at $4^{\circ} \mathrm{C}$, the antibodies were diluted using Primary Antibody Dilution Buffer (cat. no. AB64211; Abcam). The slides were washed 3 times with PBS, and incubated with the corresponding secondary antibody (1:500; cat. no. A0208; Beyotime Institute of Biotechnology) and treated with a streptavidin derivative coupled to alkaline phosphatase (cat. no. 21324; Thermo Fisher Scientific, Inc.). The sections were stained with DAB chromogen A for $5 \mathrm{~min}$ at room temperature (cat. no. P0203; Beyotime Institute of Biotechnology). Subsequently, the slides were examined at x20 magnification using an Olympus BX-UCB light microscope. 
A

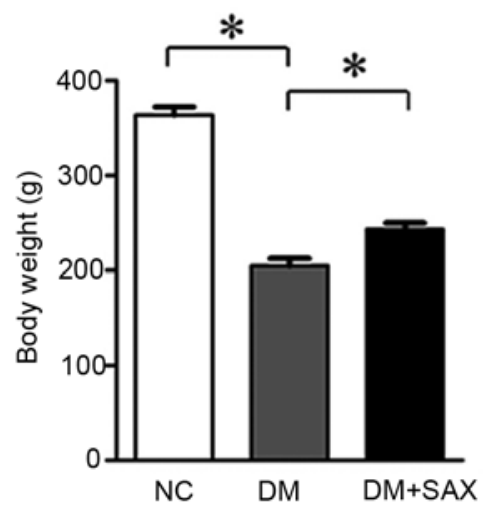

B

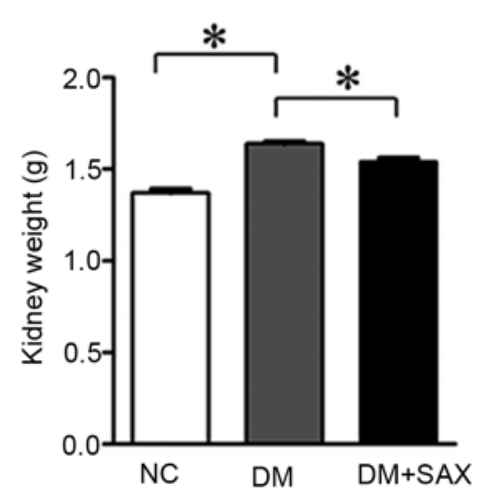

C

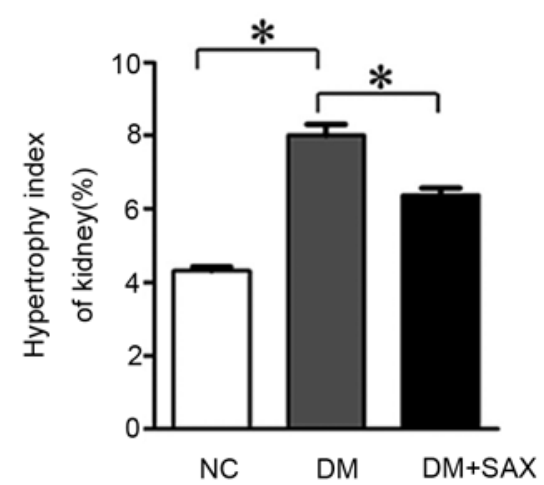

Figure 1. SAX prevents weight loss and decreases the renal weight and renal hypertrophy index in the diabetic animals. (A) Body weight, (B) kidney weight and $(C)$ hypertrophy index of the kidney of the NC, DM and DM + SAX treated rats. ${ }^{*} \mathrm{P}<0.05$. SAX, saxagliptin; DM, diabetes mellitus; NC, normal control.

Flow cytometry. To detect the induction of apoptosis, treated HK2 cells were washed twice with cold PBS, incubated with $10 \mu \mathrm{g} / \mathrm{ml}$ Annexin V-FITC (BD) for $15 \mathrm{~min}$ at room temperature, and subsequently propidium iodide (PI; $10 \mu \mathrm{l}$ ) was added and incubated for $5 \mathrm{~min}$ at room temperature. The samples were analyzed using CytoFlex software (version 2.0; Beckman Coulter, Inc.).

Western blotting. Tissues and cells were lysed using RIPA lysis buffer [150 mM NaCl, $1 \%$ NP-40, 0.1\% SDS, $0.5 \%$ deoxycholic sodium salt, $50 \mathrm{mM}$ Tris $\mathrm{HCl}(\mathrm{pH}$ 7.4), $2 \mathrm{mM}$ EDTA, $2 \mathrm{mM} \mathrm{Na} \mathrm{VO}_{4}, 10 \mathrm{mM} \mathrm{NaF}$ and one tablet of Roche Complete inhibitor cocktail (Roche Diagnostics) per $30 \mathrm{ml}]$. The protein concentration was measured using a BCA protein assay kit. Equal amounts of protein $(50 \mu \mathrm{g})$ from each sample were separated on a $10 \%$ Nupage gel, transferred to the nitrocellulose membranes and immunoblotted with primary antibodies overnight at $4^{\circ} \mathrm{C}$. All the primary antibodies were used at a 1:1,000 dilution (antibody: Dilution buffer). The membranes were washed with Tris-buffered saline containing Tween-20 $(0.15 \%$, TBS-T), incubated with horseradish peroxidase-conjugated secondary antibody $(1: 5,000)$ for $2 \mathrm{~h}$ at room temperature, and washed 3 times with TBS-T. The blots were visualized using Enhanced Chemiluminescence Plus reagent (EMD Millipore) and imaged using a GE gel imaging AI600 (Amersham Imager 600; GE Healthcare).

Statistical analysis. Experiments were repeated at least three times and similar results were obtained. Quantitative results are expressed as the mean \pm the standard error of the mean. The differences between two groups were evaluated using a Student's t-test, and comparisons between multiple groups were performed using a one-way ANOVA followed by Bonferroni's correction. $\mathrm{P}<0.05$ was considered to indicate a statistically significant difference.

\section{Results}

SAX partially reverses DM-associated weight loss and renal hypertrophy. The weights of the mice in each group did not differ significantly at the start of the study or when the animals were 10 weeks old (data not shown). At the 30 -week old period, DM animals exhibited a $43.6 \%$ lower weight compared with the control animals $(\mathrm{P}<0.01)$. This weight loss was reversed (by $18.6 \%$ ) in the DM + SAX group compared with DM animals (Fig. 1A).

At the start of the study, all three animal groups exhibited similar renal weights and similar values in the renal hypertrophy index (data not shown). At 30 weeks, the DM group exhibited a $19.7 \%$ increase in renal weight and a $84.6 \%$ higher renal hypertrophy index compared with those of the control group (both $\mathrm{P}<0.01$ ), and these increases were reversed (by 5.71 and $20.2 \%$ in the DM + SAX group compared with DM animals; Fig. 1B and C). These results suggested that SAX could protect against DM-induced weight loss and renal hypertrophy.

SAX decreases glucose levels in diabetic rats. At the beginning of the study, the three animal groups exhibited similar blood glucose levels (data not shown). At 30 weeks of age, DM animals had significantly higher blood glucose levels compared with the control animals (Fig. 2A). DM + SAX animals had significantly lower blood glucose levels compared with the DM animals (Fig. 2A). These results suggested that SAX reduced glucose levels in diabetic animals.

SAX improves kidney function in diabetic rats. Kidney function of DM rats in the presence or absence of SAX was assessed based on the blood urea nitrogen (BUN) levels, serum creatinine (Scr) levels and urinary albumin excretion rate. The levels of all three indices were significantly higher in the DM group compared with those observed in the control group $(\mathrm{P}<0.05)$, whereas they were significantly lower in the $\mathrm{DM}+\mathrm{SAX}$ group compared with the DM group (Fig. 2B-D). These results suggest that SAX improves kidney function in diabetes.

SAX reduces kidney lesions in diabetic rats. The tubulointerstitial and glomerular lesions in the three animal groups were analyzed using H\&E, Masson's trichrome, PAS and Sirus red staining. At 30 weeks of age, DM rats exhibited mesangial expansion, nodular sclerosis, glomerular atrophy and glomerulosclerosis, whereas these 
A

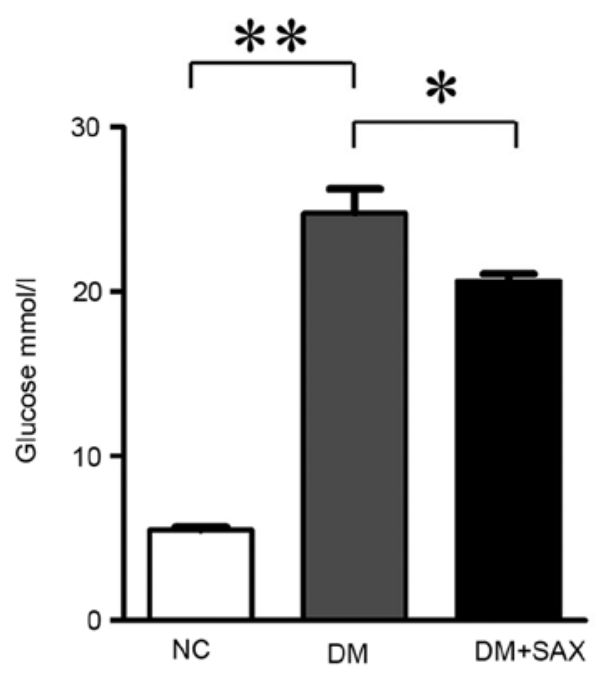

C

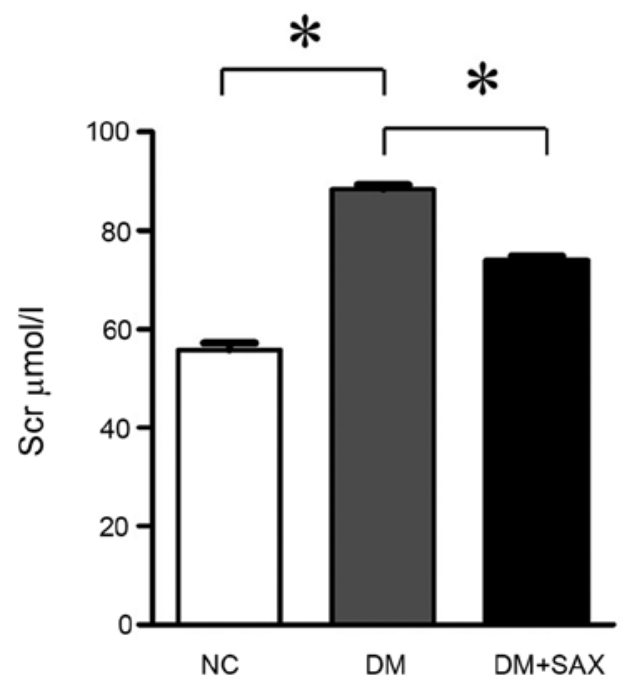

B
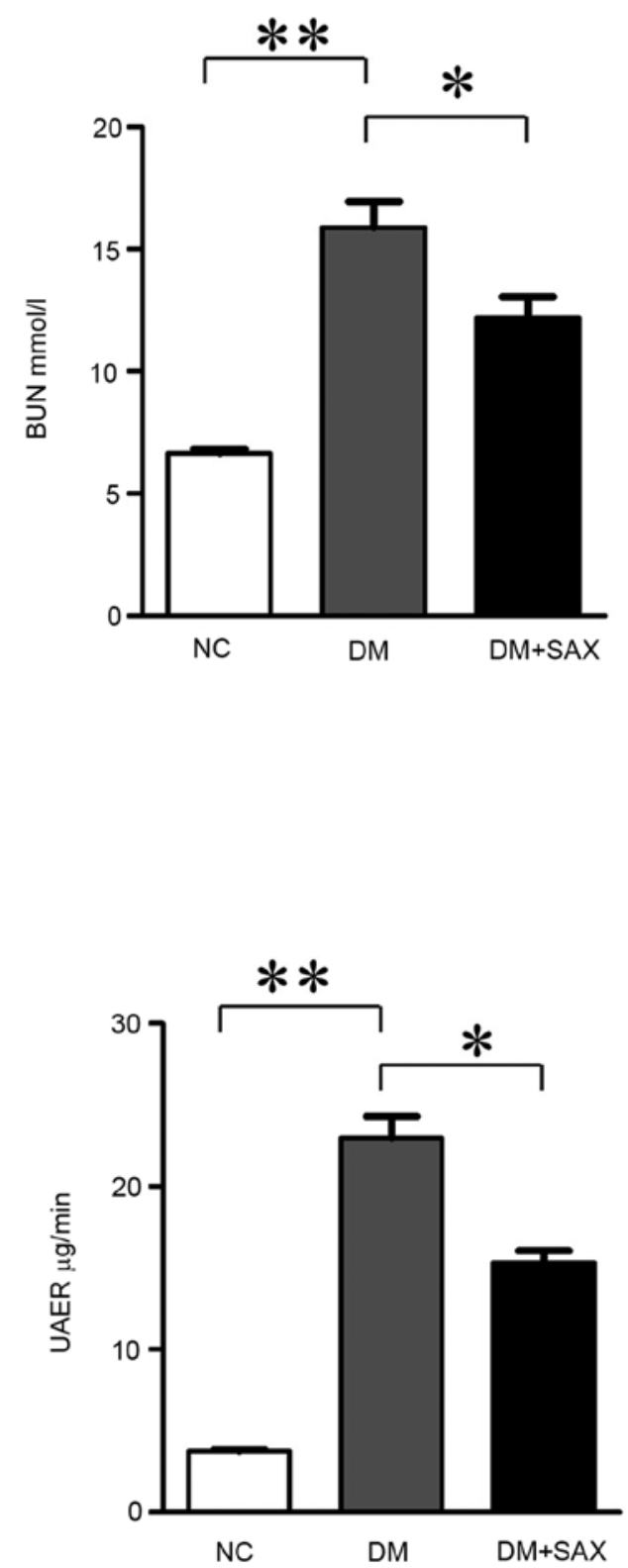

Figure 2. SAX decreases glucose levels and ameliorates kidney function in the diabetic animals. (A) Plasma glucose, (B) plasma BUN, (C) plasma Scr and (D) UAER in the NC, DM and DM + SAX treated rats. "P $<0.05,{ }^{* *} \mathrm{P}<0.01$. SAX, saxagliptin; BUN, blood urea nitrogen; Scr, serum creatinine; UAER, urinary albumin excretion rates; DM, diabetes mellitus; NC, normal control.

pathological conditions were not observed in the control animals (Fig. 3A). DM rats exhibited tubular degeneration, tubular basement membrane irregularity, interstitial fibrosis and tubular atrophy (Figs. 3B-D and S1A-C). Treatment with SAX was associated with a reduced incidence of severe fibrosis and global glomerulosclerosis, a lower number of hyaline cylinders and reduced formation of irregular basement membranes, tubular degeneration, interstitial fibrosis and tubular atrophy compared with the corresponding indices in the DM group (Figs. 3 and S1A-C; $\mathrm{P}<0.05$ ). These results suggest that $\mathrm{SAX}$ reduces the formation and severity of kidney lesions in diabetic animals.

SAX protects against $H G$-induced apoptosis. HG levels have been reported to increase apoptosis in cultured glomerular epithelial cells. Hochest 33258 staining showed that the induction of HK2 cell apoptosis was caused by HG levels. This effect was reversed with SAX treatment (Fig. 4A and B). Furthermore, flow cytometry analysis confirmed that the induction of HK2 cell apoptosis (Annexin V and PI double-positive cells) was decreased significantly in the SAX treated cells (Fig. 4C and D). These results indicate that SAX ameliorates HK2 cell apoptosis induced by HG.

SAX protects against kidney injury from DM-induced apoptosis via the caspase 3/PARP-1 pathway. Since PARP-1 is an important regulator of apoptosis, the expression levels of cleaved-caspase 3, caspase 3, cleaved-PARP-1, PARP-1, Bax and $\mathrm{Bcl} 2$ were examined. The induction of $\mathrm{DM}$ in the animal models resulted in upregulation of the expression levels of cleaved-caspase 3 , caspase 3 , cleaved-PARP-1, PARP-1 and the ratio of $\mathrm{Bax} / \mathrm{Bcl} 2$. In the presence of $\mathrm{SAX}$, this effect 
A
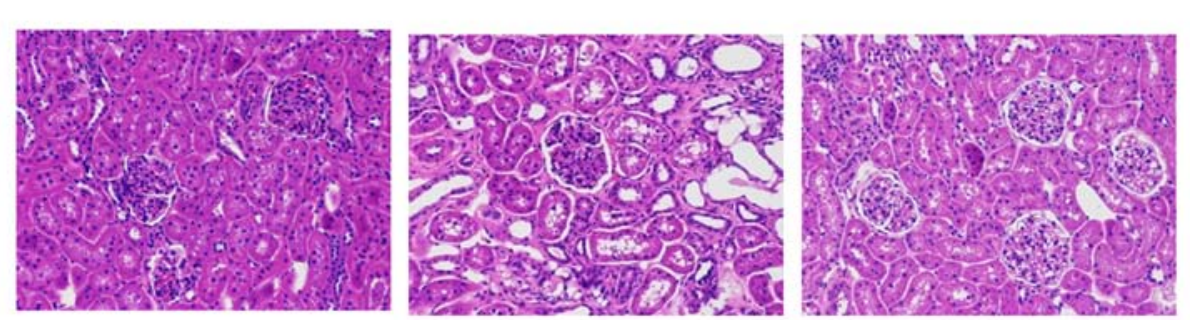

H\&E

B
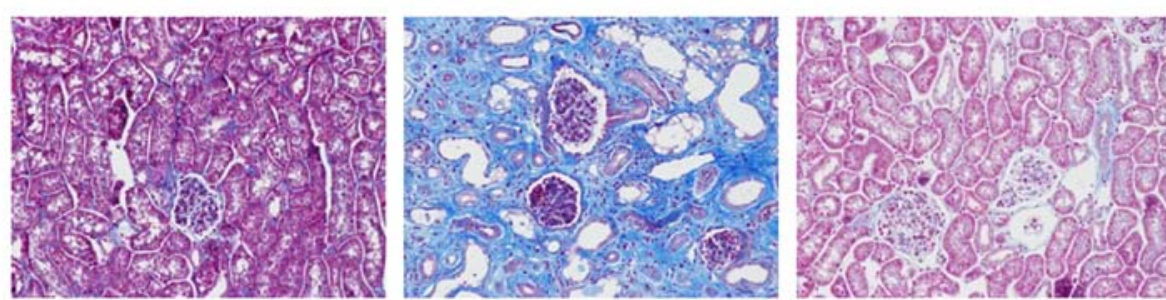

PAS

C
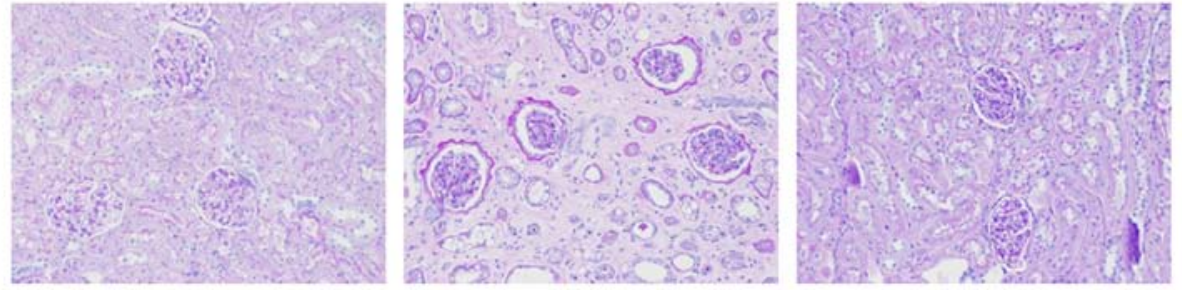

Masson's

trichrome

D

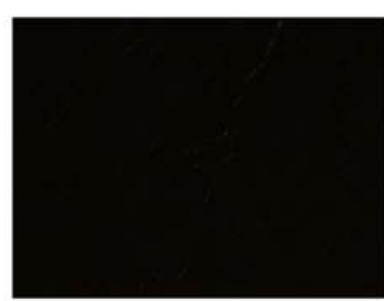

NC

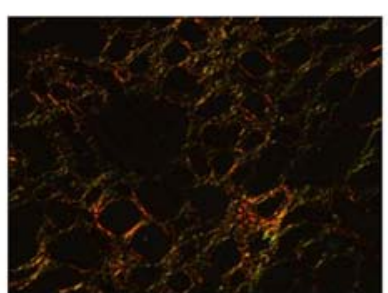

DM

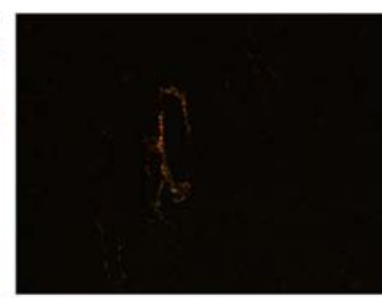

$D M+S A X$

Figure 3. SAX ameliorates kidney lesions in the diabetic animals as detected by (A) H\&E, (B) PAS, (C) Masson's trichrome and (D) Sirus red staining in the NC, DM and DM + SAX treated rats. SAX, saxagliptin; H\&E, hematoxylin and eosin; DM, diabetes mellitus; NC, normal control.

A
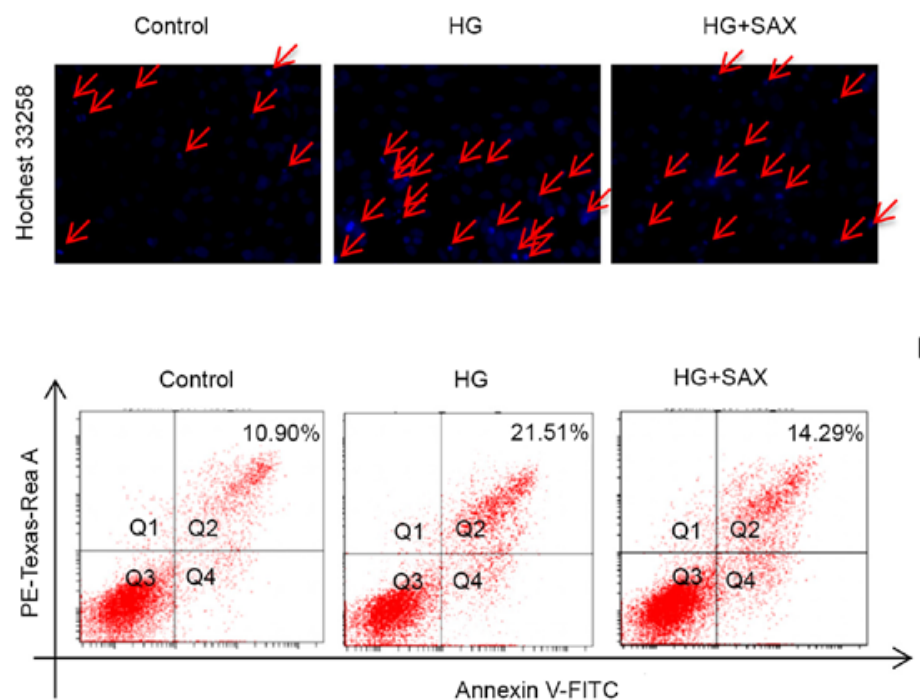

B

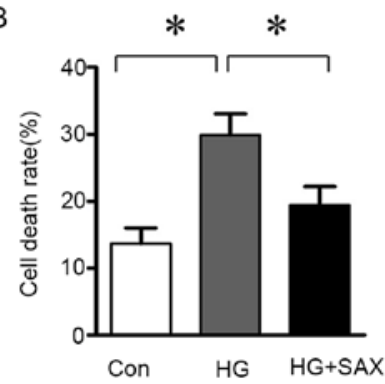

D

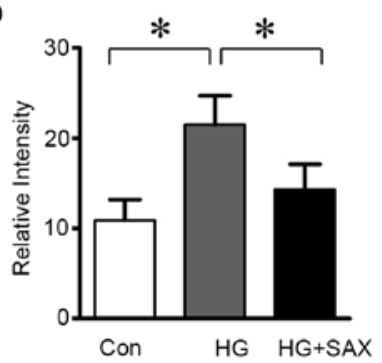

Figure 4. SAX ameliorates apoptosis in HG treated HK2 cells. (A) Induction of apoptosis was detected by Hochest 33258 fluorescent staining in the Con, HG and HG + SAX groups and (B) quantification of Hochest 33258 fluorescent staining. The red arrows indicate the apoptotic cells. (C) Apoptosis was detected by flow cytometry assays in the Con group, HG group, and the HG + SAX group. (D) Quantification of the Hochest 33258 fluorescent staining of HK2 cells. "P<0.05. SAX, Saxagliptin; Con, control; HG, high glucose; HG + SAX, high glucose with SAX. 
A

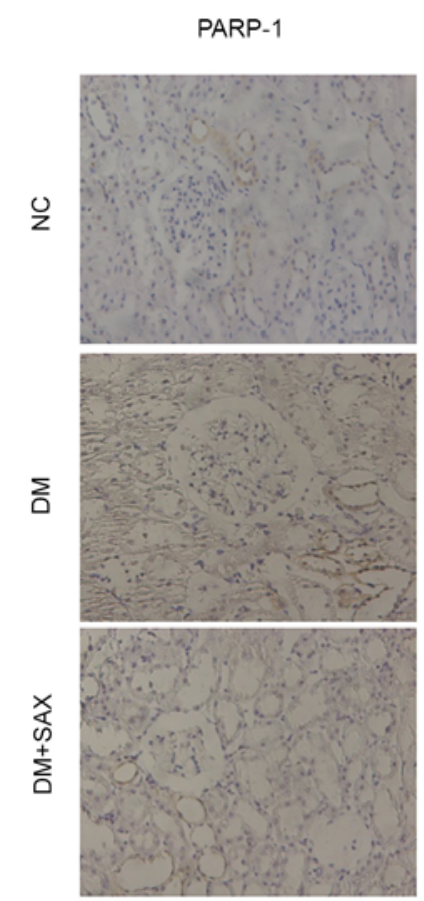

C

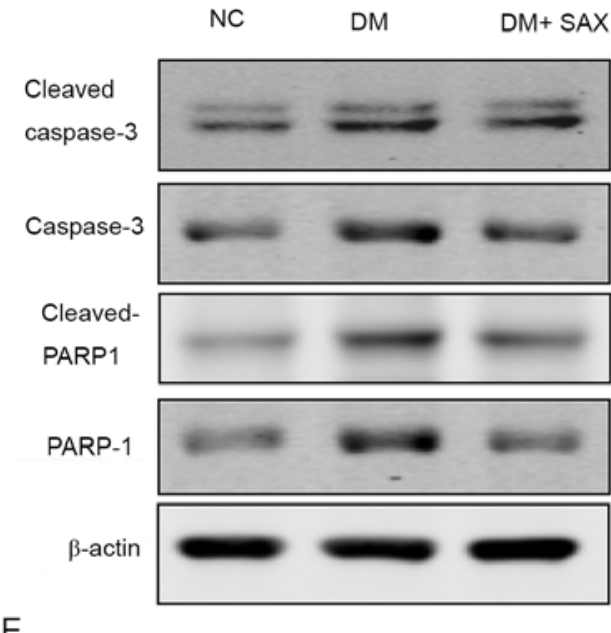

E

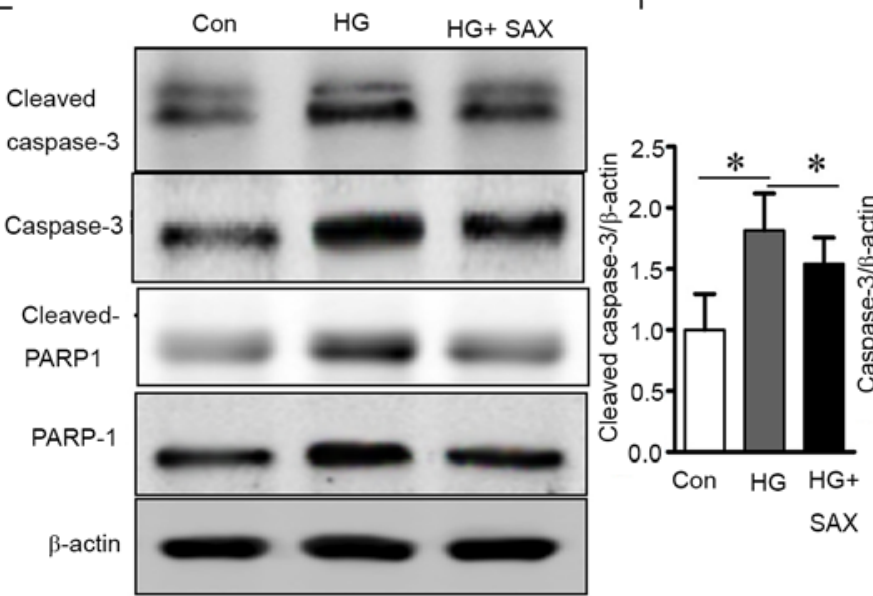

Caspase-3

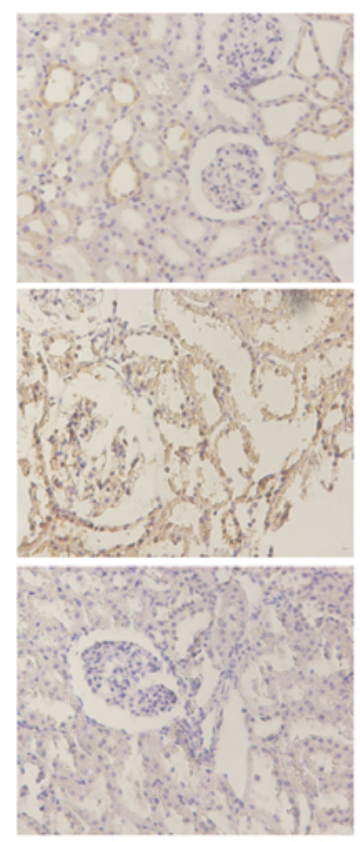

D
B
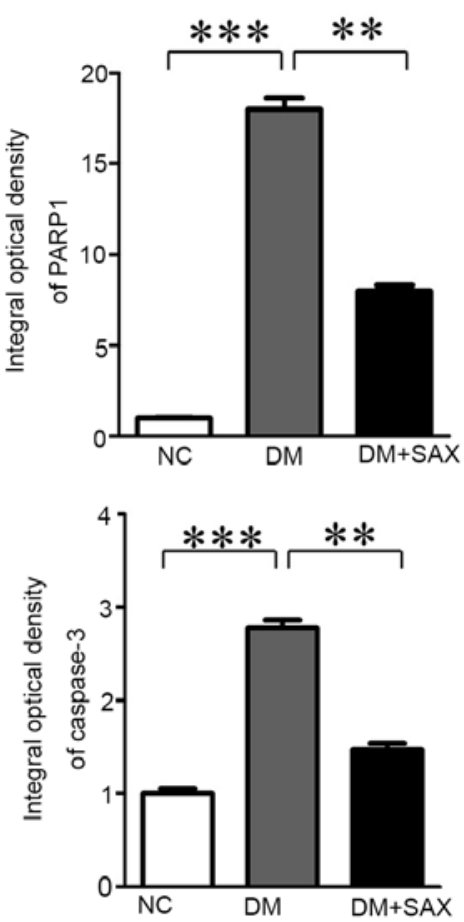
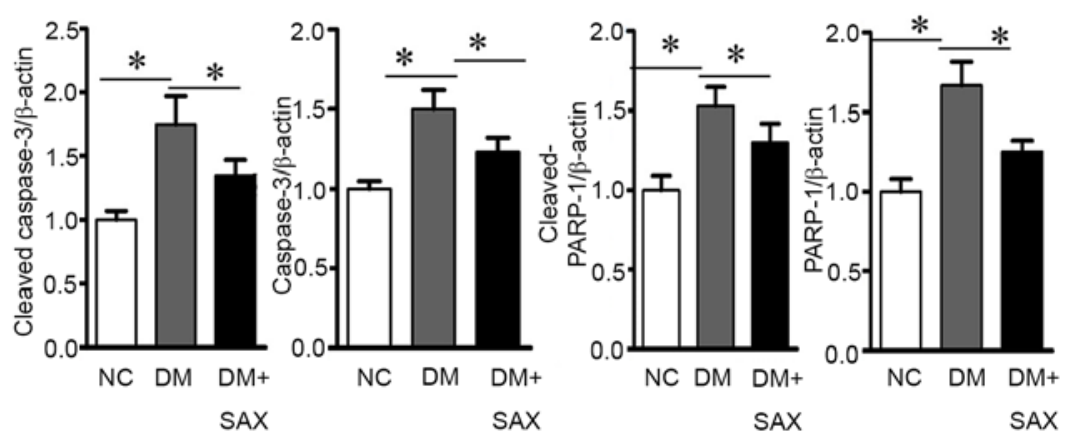

Figure 5. SAX protects the diabetic kidney against apoptotic cell death induced by diabetes and HG. (A) Caspase 3 and PARP-1 protein expression in rats in the NC, DM and DM + SAX groups as determined by immunohistochemistry. (B) Quantification of the expression levels of caspase 3 and PARP-1 protein based on the immunohistochemistry analysis. (C) Expression levels of cleaved-caspase 3, caspase 3, cleaved-PARP-1 and PARP-1 protein detection in the NC, DM, and DM + SAX treated groups were determined by western blot analysis, and (D) quantification of the western blot data. (E) Western blot analysis of cleaved-caspase 3, caspase 3, cleaved-PARP-1 and PARP-1 in the control, HG treated and HG + SAX treated HK2 cells. (F) Densitometry analysis of the western blot data. ${ }^{*} \mathrm{P}<0.05,{ }^{* *} \mathrm{P}<0.01,{ }^{* * * *} \mathrm{P}<0.001$. SAX, saxagliptin; HG, high glucose; PARP-1, poly (ADP-ribose) polymerase-1; NC, normal control; DM, diabetes mellitus; T2DM, diabetes mellitus type 2; HG + SAX, high glucose with SAX. 
A

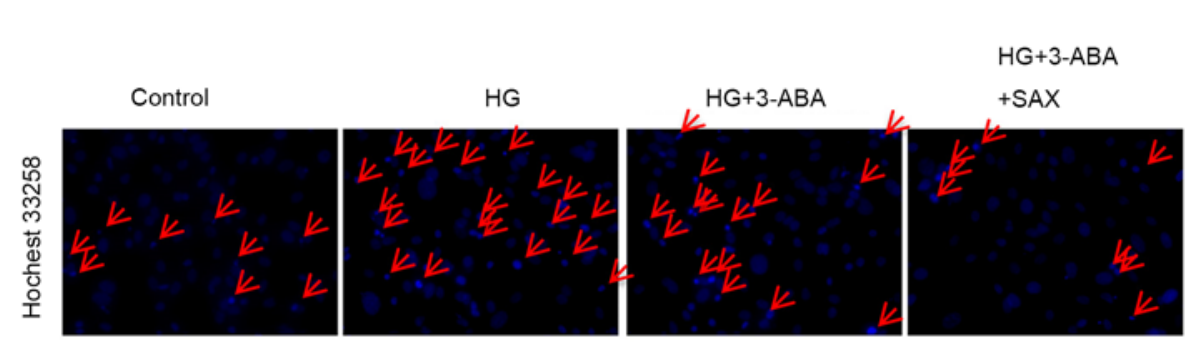

C

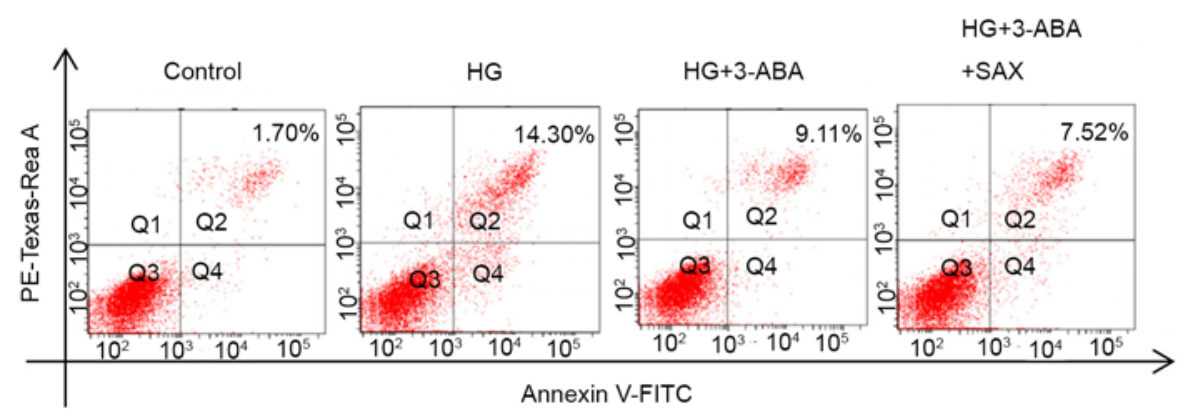

B
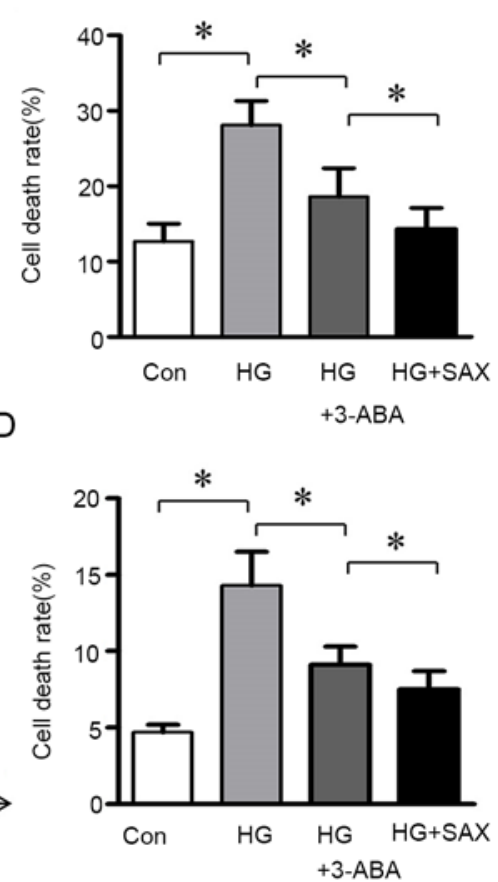

Figure 6. SAX ameliorates the induction of apoptosis in HG induced HK2 cells by inhibiting PARP-1 activity. (A) Induction of apoptosis was detected by Hochest 33258 fluorescent staining in the Con, HG, HG + 3-ABA (PARP-1 inhibitor) and HG + 3-ABA + SAX groups and (B) quantification of Hochest 33258 fluorescent staining. The red arrows indicate the apoptotic cells. (C) Induction of apoptosis was detected by flow cytometry assays in the Con, HG and $\mathrm{HG}+\mathrm{SAX}$ groups and (D) Hochest 33258 fluorescent staining was quantified. ${ }^{\text {P }<0.05 . ~ S A X, ~ s a x a g l i p t i n ; ~ H G, ~ h i g h ~ g l u c o s e ; ~ P A R P-1, ~ p o l y ~(A D P-r i b o s e) ~}$ polymerase-1; Con, control; ABA, 3-Aminobenzamide; HG + SAX, high glucose with SAX.

was partially reversed (Figs. 5A-D and S2A). Moreover, SAX partially reversed the upregulation of cleaved caspase 3 , caspase 3, cleaved-PARP-1, PARP-1 and the ratio of Bax/Bcl2 induced by $\mathrm{HG}$ treatment in the HK2 cells (Figs. 5E and F and S2B). 3-ABA inhibited the induction of apoptosis by HG. SAX partially increased the inhibitory effects of 3-ABA on apoptosis in HK2 cells (Fig. 6A-D). These results suggested that SAX inhibited apoptosis via the caspase 3/PARP-1 pathway, and thereby protected the tissues from the effects of diabetic nephropathy.

\section{Discussion}

In the present study, SAX was shown to protect against the pathological effects associated with diabetic nephropathy. In the rat model of streptozocin-induced DM, SAX reduced glycemia, tubulointerstitial and glomerular damage, and improved kidney function. These therapeutic changes were associated with downregulation of the expression of apoptotic genes, such as cleaved-caspase 3 and cleaved-PARP-1. Moreover, the present study demonstrated that SAX could downregulate the ratio of $\mathrm{Bax} / \mathrm{Bcl} 2$. The findings suggested that long-term SAX administration could protect against diabetic nephropathy and provide an explanation of the mechanisms of apoptosis inhibition.

The incidence of diabetic nephropathy is continuously increasing $(31,32)$ and is considered to be the primary cause of chronic kidney disease and end-stage renal failure (33). Diabetic nephropathy is a chronic, complex disease that gradually leads to glomerular hypertrophy, glomerular basement membrane thickening, collagen deposition, interstitial fibrosis aggravation, renal tubular atrophy and renal function disorder $(34,35)$. $\mathrm{HG}$ alters the microenvironment of each cell, and in turn leads to the activation of the apoptotic pathway $(10,36)$.

By inhibiting DPP-4 and slowing down GLP-1 degradation, SAX can partially reverse the reduction in the incretin effect in the absence of hypoglycemia (37). SAX can correct glucose metabolism disorders and prevent kidney injury (38). Its cytoprotective effects have been noted on several types of cells, including cells in the pancreas, heart and retina (39). The present study identified a novel mechanism by which SAX could restore existing kidney dysfunction. SAX significantly decreased the DM-induced increase in weight and kidney hypertrophy. It also ameliorated DM-induced structural kidney injury and fibrosis, as shown by H\&E staining of tissue slices, and it reduced DM-induced glycogen accumulation in the kidney. The results provide a plausible explanation by which these beneficial alterations are mediated; downregulation of the expression of the pro-apoptotic proteins. In the rat model used, DM increased the expression of caspase 3, which cleaved PARP-1, causing DNA damage and loss of DNA repair ability $(40,41)$; SAX partially reversed these effects. Similarly, the DPP-4 inhibitor linagliptin ameliorated DM-induced kidney damage by inhibiting apoptosis (5).

In conclusion, the results showed that SAX could improve kidney function in diabetic rats in part by downregulating caspase 3 expression and thereby inhibiting the pro-apoptotic cleavage of PARP-1. The results are in agreement with those reported in previous studies assessing the effects of SAX. This compound may thus serve as a 
promising renoprotective therapy against the development and progression of diabetic nephropathy and may serve as a novel therapeutic option for the prevention and treatment of diabetic nephropathy.

\section{Acknowledgements}

Not applicable.

\section{Funding}

This work was supported by the Youth Fund of the Second Hospital of Shandong University (grant no. Y2013010015), PhD Foundation of Shandong Natural Science Foundation (grant no. ZR2018BH010), Key Research and Development Project in Shandong (grant no. 2017G006027), Shandong Medical and Health Science and Technology Development Program Project (grant no. 2013WS0243). Shandong Provincial Natural Science Foundation (grant no.ZR2016HP01) and China Postdoctoral Science Foundation (grant no. 2018M642673).

\section{Availability of data and materials}

The datasets used and/or analyzed during the current study are available from the corresponding author on reasonable request.

\section{Authors' contributions}

$\mathrm{XX}, \mathrm{XW}$ and QL designed and performed experiments, and wrote the manuscript. SG, YL, JK and $\mathrm{ZH}$ performed the experiments. XW and QL confirm the authenticity of all the raw data. XX, JK, XW and QL acquired, analyzed and interpreted the data and were involved in writing, reviewing and editing the manuscript, as well as supervision. All authors have read and approved the final manuscript.

\section{Ethics approval and consent to participate}

All animal protocols were reviewed and approved by the Animal Care and Use Committee of Shandong University.

\section{Patient consent for publication}

Not applicable.

\section{Competing interests}

The authors declare that they have no competing interests.

\section{References}

1. Giacco F and Brownlee M: Oxidative stress and diabetic complications. Circ Res 107: 1058-1070, 2010.

2. Reidy K, Kang HM, Hostetter T and Susztak K: Molecular mechanisms of diabetic kidney disease. J Clin Invest 124: 2333-2340, 2014.

3. Lindblom R, Higgins G, Coughlan M and de Haan JB: Targeting mitochondria and reactive oxygen species-driven pathogenesis in diabetic nephropathy. Rev Diabet Stud 12: 134-156, 2015.

4. Dai H, Liu Q and Liu B: Research progress on mechanism of podocyte depletion in diabetic nephropathy. J Diabetes Res 2017: 2615286, 2017.
5. Marques C, Mega C, Goncalves A, Rodrigues-Santos P, Teixeira-Lemos E, Teixeira F, Fontes-Ribeiro C, Reis F and Fernandes R: Sitagliptin prevents inflammation and apoptotic cell death in the kidney of type 2 diabetic animals. Mediators Inflamm 2014: 538737, 2014

6. Kanasaki K, Shi S, Kanasaki M, He J, Nagai T, Nakamura Y, Ishigaki Y, Kitada M, Srivastava SP and Koya D: Linagliptin-mediated DPP-4 inhibition ameliorates kidney fibrosis in streptozotocin-induced diabetic mice by inhibiting endothelial-to-mesenchymal transition in a therapeutic regimen. Diabetes 63: 2120-2131, 2014.

7. Rivero A, Mora C, Muros M, García J, Herrera H and Navarrogonzález JF: Pathogenic perspectives for the role of inflammation in diabetic nephropathy. Clin Sci (Lond) 116: 479-492, 2009.

8. Sanchez-Niño MD, Benito-Martin A and Ortiz A: New paradigms in cell death in human diabetic nephropathy. Kidney Int 78: 737-744, 2010.

9. Wagener FA, Dekker D, Berden JH, Scharstuhl A and van der Vlag J: The role of reactive oxygen species in apoptosis of the diabetic kidney. Apoptosis 14: 1451-1458, 2009.

10. Volpe CMO, Villar-Delfino PH, Dos Anjos PMF and Nogueira-Machado JA: Cellular death, reactive oxygen species (ROS) and diabetic complications. Cell Death Dis 9: 119, 2018.

11. Ma Y, Chen F, Yang S, Chen B and Shi J: Protocatechuic acid ameliorates high glucose-induced extracellular matrix accumulation in diabetic nephropathy. Biomed Pharmacother 98: 18-22, 2018.

12. Gray SP and Jandeleit-Dahm K: The pathobiology of diabetic vascular complications-cardiovascular and kidney disease. J Mol Med (Berl) 92: 441-452, 2014.

13. Szabó C, Biser A, Benko R, Böttinger E and Suszták K: Poly(ADP-ribose) polymerase inhibitors ameliorate nephropathy of type 2 diabetic Leprdb/db mice. Diabetes 55: 3004-3012, 2006.

14. Shevalye H, Maksimchyk Y, Watcho P and Obrosova IG: Poly(ADP-ribose) polymerase-1 (PARP-1) gene deficiency alleviates diabetic kidney disease. Biochim Biophys Acta 1802: 1020-1027, 2010.

15. Chao LK, Chang WT, Shih YW and Huang JS: Cinnamaldehyde impairs high glucose-induced hypertrophy in renal interstitial fibroblasts. Toxicol Appl Pharmacol 244: 174-180, 2010.

16. Lau GJ, Godin N, Maachi H, Lo CS, Wu SJ, Zhu JX, Brezniceanu ML, Chénier I, Fragasso-Marquis J, Lattouf JB, et al: Bcl-2-modifying factor induces renal proximal tubular cell apoptosis in diabetic mice. Diabetes 61: 474-484, 2012.

17. Covington MD and Schnellmann RG: Chronic high glucose downregulates mitochondrial calpain 10 and contributes to renal cell death and diabetes-induced renal injury. Kidney Int 81: 391-400, 2012.

18. Sharkovska Y, Reichetzeder C, Alter M, Tsuprykov O, Bachmann S, Secher T, Klein T and Hocher B: Blood pressure and glucose independent renoprotective effects of dipeptidyl peptidase- 4 inhibition in a mouse model of type- 2 diabetic nephropathy. J Hypertens 32: 2211-2223, 2014.

19. Borzi AM, Condorelli G, Biondi A, Basile F, Vicari ESD, Buscemi C, Luca S and Vacante M: Effects of vildagliptin, a DPP-4 inhibitor, in elderly diabetic patients with mild cognitive impairment. Arch Gerontol Geriatr 84: 103896, 2019.

20. Shi S, Koya D and Kanasaki K: Dipeptidyl peptidase-4 and kidney fibrosis in diabetes. Fibrogenesis Tissue Repair 9: 1, 2016.

21. Itou M, Kawaguchi T, Taniguchi E and Sata M: Dipeptidyl peptidase-4: A key player in chronic liver disease. World $\mathrm{J}$ Gastroenterol 19: 2298-2306, 2013.

22. Shirakawa J, Fujii H, Ohnuma K, Sato K, Ito Y, Kaji M, Sakamoto E, Koganei M, Sasaki H, Nagashima Y, et al: Diet-induced adipose tissue inflammation and liver steatosis are prevented by DPP-4 inhibition in diabetic mice. Diabetes 60: 1246-1257, 2011.

23. Nistala R, Habibi J, Aroor A, Sowers JR, Hayden MR, Meuth A, Knight W, Hancock T, Klein T, Demarco VG and Whaley-Connell A: DPP4 inhibition attenuates filtration barrier injury and oxidant stress in the zucker obese rat. Obesity (Silver Spring) 22: 2172-2179, 2014.

24. Groop PH, Cooper ME, Perkovic V, Emser A, Woerle HJ and Eynatten M: Linagliptin lowers albuminuria on top of recommended standard treatment in patients with type 2 diabetes and renal dysfunction. Diabetes Care 36: 3460-3468, 2013. 
25. Ommen ES, Xu L, O'Neill EA, Goldstein BJ, Kaufman KD and Engel SS: Comparison of treatment with sitagliptin or sulfonylurea in patients with type 2 diabetes mellitus and mild renal impairment: A post hoc analysis of clinical trials. Diabetes Ther 6: 29-40, 2015

26. Lim S, Han KA, Yu J, Chamnan P, Kim ES, Yoon KH, Kwon S, Moon MK, Lee KW, Kim DJ, et al: Efficacy and safety of initial combination therapy with gemigliptin and metformin compared with monotherapy with either drug in patients with type 2 diabetes: A double-blind randomized controlled trial (INICOM study). Diabetes Obes Metab 19: 87-97, 2017.

27. Hardy G: Saxagliptin demonstrates no increased risk for cardiovascular death, heart attack or stroke in the SAVOR cardiovascular outcomes trial. Cardiovasc J Afr 24: 290, 2013.

28. Ott C, Raff U, Schmidt S, Kistner I, Friedrich S, Bramlage P, Harazny JM and Schmieder RE: Effects of saxagliptin on early microvascular changes in patients with type 2 diabetes. Cardiovasc Diabetol 13: 19, 2014.

29. Roy D, Chadwick KD, Tatarkiewicz K, LaCerte C, Bergholm AM, Brodie T, Mangipudy RS, Parkes D, Graziano MJ and Reilly TP: The glucagon-like peptide-1-based therapeutics exenatide and saxagliptin did not cause detrimental effects on the pancreas in mice, rats, dogs and monkeys. Diabetes Obes Metab 16: 910-921, 2014.

30. Lee TI, Kao YH, Tsai WC, Chung CC, Chen YC and Chen YJ: HDAC inhibition modulates cardiac PPARs and fatty acid metabolism in diabetic cardiomyopathy. PPAR Res 2016: 5938740, 2016

31. Bae EJ: DPP-4 inhibitors in diabetic complications: Role of DPP-4 beyond glucose control. Arch Pharm Res 39: 1114-1128, 2016.

32. Xiao T, Guan X, Nie L, Wang S, Sun L, He T, Huang Y, Zhang J, Yang $\mathrm{K}$, Wang $\mathbf{J}$ and Zhao J: Rapamycin promotes podocyte autophagy and ameliorates renal injury in diabetic mice. Mol Cell Biochem 394: 145-154, 2014.
33. Lv M, Chen Z, Hu G and Li Q: Therapeutic strategies of diabetic nephropathy: Recent progress and future perspectives. Drug Discov Today 20: 332-346, 2015.

34. Jefferson JA, Shankland SJ and Pichler RH: Proteinuria in diabetic kidney disease: A mechanistic viewpoint. Kidney Int 74: 22-36, 2008.

35. Flyvbjerg A: The role of the complement system in diabetic nephropathy. Nat Rev Nephrol 13: 311-318, 2017.

36. Xiao L, Wang M, Yang S, Liu F and Sun L: A glimpse of the pathogenetic mechanisms of $\mathrm{Wnt} / \beta$-catenin signaling in diabetic nephropathy. Biomed Res Int 2013: 987064, 2013.

37. Schernthaner G, Durán-Garcia S, Hanefeld M, Langslet G, Niskanen L, Östgren CJ, Malvolti E and Hardy E: Efficacy and tolerability of saxagliptin compared with glimepiride in elderly patients with type 2 diabetes: A randomized, controlled study (GENERATION). Diabetes Obes Metab 17: 630-638, 2015.

38. Scheen AJ: DPP-4 inhibitors in the management of type 2 diabetes: A critical review of head-to-head trials. Diabetes Metab 38: 89-101, 2012.

39. Garg K, Tripathi CD and Kumar S: Clinical review of sitagliptin: A DPP-4 inhibitor. J Assoc Physicians India 61: 645-649, 2013.

40. Cai Y, Lydic TA, Turkette T, Reid GE and Olson LK: Impact of alogliptin and pioglitazone on lipid metabolism in islets of prediabetic and diabetic zucker diabetic fatty rats. Biochem Pharmacol 95: 46-57, 2015.

41. Verzola D, Bertolotto MB, Villaggio B, Ottonello L, Dallegri F, Frumento G, Berruti V, Gandolfo MT, Garibotto G and Deferran G: Taurine prevents apoptosis induced by high ambient glucose in human tubule renal cells. J Investig Med 50: 443-451, 2002.

This work is licensed under a Creative Commons Attribution-NonCommercial-NoDerivatives 4.0 International (CC BY-NC-ND 4.0) License. 